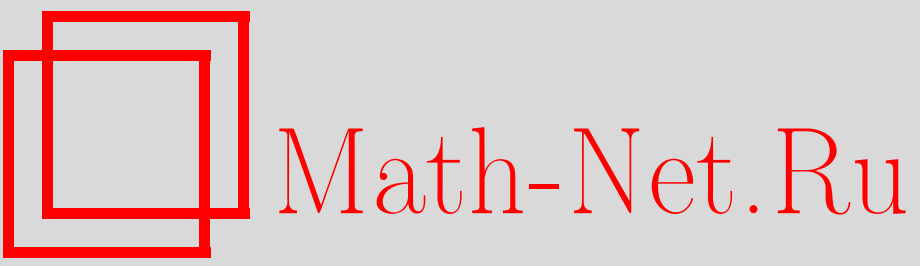

А. Х. Бикулов, Стохастические уравнения математической физики над полем p-адических чисел, ТМФ, 1999, том 119, номер 2, 249-263

DOI: https://doi.org/10.4213/tmf737

Использование Общероссийского математического портала Math-Net.Ru подразумевает, что вы прочитали и согласны с пользовательским соглашением

http://www.mathnet.ru/rus/agreement

Параметры загрузки:

IP : 54.80 .73 .141

26 апреля 2023 г., 15:11:23

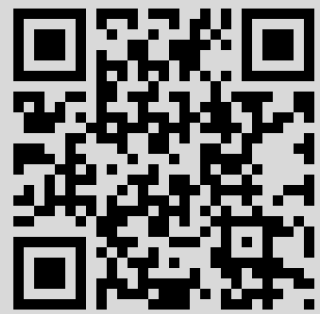


ТЕОРЕТИЧЕСКАЯ

И МАТЕМАТИЧЕСКАЯ

ФИЗИКА

Том 119, № 2

май, 1999

(C) 1999 г.

А.Х. Бикулов*

\section{СТОХАСТИЧЕСКИЕ УРАВНЕНИЯ \\ МАТЕМАТИЧЕСКОЙ ФИЗИКИ НАД ПОЛЕМ $p$-АДИЧЕСКИХ ЧИСЕЛ}

Исследованы свойства и вероятностные характеристики решений $p$-адических стохастических уравнений математической физики с псевдодифференциальным оператором Владимирова и его многомерным обобщением. Построены $p$-адические аналоги броуновского листа и броуновского движения Леви.

\section{1. ВВЕДЕНИЕ}

В последние годы проводятся интенсивные исследования по применению $p$-адического анализа в математической физике, в частности в теории случайных процессов [1].

В [2] Воловичем и автором был построен и исследован новьй случайный процесс над кольцом целых $p$-адических чисел, названный $p$-адическим броуновским движением в силу некоторых его свойств, общих с классическим броуновским движением.

В данной статье исследуются случайные поля как решения стохастических обобшенных псевдодифференциальных уравнений, являюшихся $p$-адическими аналогами уравнений математической физики, таких как волновое уравнение и уравнение Лапласа. Роль оператора дифференцирования играет псевдодифференциальный оператор Владимирова [3].

Статья организована следующим образом. Во втором разделе излагаются необходимые для дальнейшего понятия из теории случайных процессов и $p$-адического анализа.

Затем в третьем разделе вводится $p$-адическое броуновское движение на $Q_{p}$ как решение псевдодифференциального уравнения - $p$-адического аналога уравнения Ланжевена - и рассматриваются некоторые его свойства.

И наконец, в четвертом разделе построены решения стохастических уравнений на $Q_{p}^{2}$. Для псевдодифференциального оператора ( $p$-адического аналога оператора гиперболического типа) исследована задача Гурса и показано, что ее решением является $p$-адический аналог броуновского листа. Для аналога оператора Лапласа с нулевым дополни-

* Институт химической физики РАН, Москва, Россия 
тельным условием в точке $(0,0)$ найдено решение, которое мы называем $p$-адическим броуновским движением Леви.

Результаты, полученные в данной работе, могут найти применение в некоторых задачах динамики сильно неупорядоченных сред с фрустрациями, таких как спиновые стекла, белковые глобулы и т.д.

О других исследованиях $p$-адических случайных процессов см. [4-6]. В работе Кочубея [7] исследуются стохастические дифференциальные уравнения с действительным временем и $p$-адической координатой. Псевдодифференциальные уравнения параболического типа над полем $p$-адических чисел рассматривались в [8].

\section{2. НЕКОТОРЫЕ ВВОДНЫЕ ПОНЯТИЯ}

Пусть дано вероятностное пространство $(\Omega, \Sigma, \mathrm{P})$ с мерой $\mathrm{P}$ на $\sigma$-алгебре событий $\Sigma$.

Случайной величиной $\xi=\xi(\omega)$ называется $\Sigma$-измеримая вешественно- или комплекснозначная функция на $\Omega$, т.е. $\xi^{-1}(B) \in \Sigma$ для любого борелевского множества $B$ на $R$ или $C$.

В дальнейшем будем рассматривать различные случайные отображения. Напомним, что случайным отображением множества $X$ в измеримое пространство $(Y, \mathcal{B})$ называется отображение $\xi: X \times \Omega \rightarrow Y$, являюшееся при произвольном $x \in X$ измеримым отображением $(\Omega, \Sigma)$ в $(Y, \mathcal{B})$.

ОПРЕДЕЛЕНИЕ 2.1. Если $X$ - подмножество в поле $p$-адических чисел $Q_{p}\left(Q_{p}^{n}\right)$, то случайное отображение $\xi(x, \omega)$ называется $p$-адической случайной функцией ( $p$-адическим случайным полем).

$p$-Адическая случайная функция называется также $p$-адическим случайным процесcom.

По поводу определения $Q_{p}$ и $Q_{p}^{n}$ см. [1]. Напомним только, что для любого простого числа $p$ поле $Q_{p}$ является пополнением поля рациональных чисел $Q$ по $p$-адической норме $|\cdot|_{p}$. Если ненулевое рациональное число представлено в виде $x=p^{k} \cdot m / n$, где $m$ и $n$ не делятся на $p$, то $|x|_{p}=p^{-k}$. Пространство $Q_{p}^{n}=Q_{p} \times Q_{p} \times \cdots \times Q_{p}$ состоит из точек $x=\left(x_{1}, x_{2}, \ldots, x_{n}\right)$.

Пусть $\mathcal{D}=\mathcal{D}\left(Q_{p}^{n}\right)$ - пространство основных функций, т.е. комплекснозначных, локально-постоянных функций с компактным носителем $p$-адического аргумента (см. [1]).

ОПРЕДЕЛЕНИЕ 2.2. $p$-Адической обобшенной случайной функцией называется случайное отображение $\xi: \mathcal{D} \times \Omega \rightarrow C$, линейное по $\varphi \in \mathcal{D}$, если для любого $B \subset \mathcal{B}$ и фиксированного $\varphi \in \mathcal{D}$ множество $\{\omega \in \Omega: \xi(\varphi, \omega) \subset B\}$ содержится в $\Sigma$.

В случае, если пространство $\mathcal{D}$ состоит из функций одного переменного, соответствуюшая обобшенная случайная функция называется $p$-адическим обобщенным случайным процессом. Если же пространство $\mathcal{D}$ является пространством функций многих переменных, $\xi$ называется $p$-адическим обобшенным случайным полем. 
Основное содержание статьи можно охарактеризовать как исследование граничных задач для обобщенных стохастических псевдодифференциальных уравнений вида

$$
\mathbf{A} \Psi=\Phi
$$

где $\Phi$ - p-адический белый шум, которой определен ниже, и $\mathbf{A}$ - псевдодифференциальный оператор (над полем $p$-адических чисел) в открытом множестве $O \subset Q_{p}^{n}$, т.е. оператор вида

$$
(\mathbf{A} \psi)(x)=\int_{Q_{p}^{n}} a(\xi, x) \tilde{\psi}(\xi) \chi(-\xi \cdot x) d^{n} \xi, \quad x \in O
$$

действуюший на комплекснозначные функции $\psi(x) p$-адического аргумента $x \in O$. Здесь функция $\psi$ предполагается заданной на всем пространстве $Q_{p}^{n}$, вне $O$ она продолжена нулем, $\tilde{\psi}(\xi)$ - ее преобразование Фурье:

$$
\tilde{\psi}(\xi)=\int_{O} \psi(x) \chi(\xi \cdot x) d^{n} x
$$

где $\xi \cdot x=\xi_{1} x_{1}+\cdots+\xi_{n} x_{n}$ и $\chi-$ аддитивный характер $\chi_{p}$ группы $Q_{p}[1]$. Функция $a(\xi, x)$, $\xi \in Q_{p}^{n}, x \in O$, назьвается символом оператора А. В случае $n=1$ и $a(\xi, x)=|\xi|_{p}^{\alpha}$, $\alpha \in R$, псевдодифференциальный оператор $A$ называется оператором Владимирова и обозначается $\mathbf{D}^{\alpha}$ (см. $\left.[1,3]\right)$. По поводу $p$-адического анализа мы отсылаем к книге [1].

\section{3. $p$-АДИЧЕСКОЕ БРОУНОВСКОЕ ДВИЖЕНИЕ НА $Q_{p}$}

В этом разделе построено и исследовано $p$-адическое броуновское движение на $Q_{p}$, которое получено как решение стохастического псевдодифференциального уравнения аналога уравнения Ланжевена. Сначала докажем его сушествование, а затем дадим определение. $p$-А дическое броуновское движение на $Z_{p}$ было построено в [2].

Пусть даны вероятностное пространство $(\Omega, \Sigma, \mathrm{P})$ и измеримое пространство $(C, \mathcal{B})$, где $C$ - поле комплексных чисел, а $\mathcal{B}$ - $\sigma$-алгебра борелевских подмножеств из $C$. Пусть дан $p$-адический обобщенный случайный процесс

$$
\Phi(\varphi ; \omega)=\langle\Phi(t ; \omega), \varphi(t)\rangle, \quad \varphi \in \mathcal{D}\left(Q_{p}\right), \quad \omega \in \Omega
$$

ОПРЕДЕЛЕНИЕ 3.1. Обобщенньй случайный процесс $\left\{\Phi(\varphi ; \omega), \varphi \in \mathcal{D}\left(Q_{p}\right)\right\}$ называется гауссовским, если для любого конечного набора функций $\varphi_{1}, \varphi_{2}, \ldots, \varphi_{n}$ из $\mathcal{D}\left(Q_{p}\right)$ и любого борелевского множества $B \subset C^{n}$ мера множества $\mathcal{A}=\left\{\omega \in \Omega:\left(\Phi\left(\varphi_{1} ; \omega\right), \ldots\right.\right.$ $\left.\left.\ldots, \Phi\left(\varphi_{n} ; \omega\right)\right) \in B\right\}$ выражается формулой

$$
\mathrm{P}\{\mathcal{A}\}=\frac{\operatorname{det} \Lambda}{(2 \pi)^{n}} \int_{B} e^{-(\Lambda z, z)} d z \wedge d \bar{z}
$$


где $\Lambda$ - положительно-определенная матрица,

$$
(\Lambda z, z)=\sum_{i, j} \Lambda_{i, j} z_{i} \bar{z}_{j}
$$

- квадратичная форма, $\Lambda^{-1}=G$, где $G$ - положительно-определенная невырожденная матрица с элементами $G_{i j}=\mathrm{E} \Phi\left(\varphi_{i}\right) \bar{\Phi}\left(\varphi_{j}\right), z=\left(z_{1}, \ldots, z_{n}\right), \bar{z}=\left(\bar{z}_{1}, \ldots, \bar{z}_{n}\right), z_{k}=x_{k}+$ $i y_{k}, \bar{z}_{k}=x_{k}-i y_{k}, k=1,2,3, \ldots, n, d z \wedge d \bar{z}=2^{n} d x_{1} d y_{1} \ldots d x_{n} d y_{n}$.

ОПРЕДЕЛЕНИЕ 3.2. $p$-Адическим белым шумом называется гауссовский обобщенньй случайный процесс $\left\{\Phi(\varphi ; \omega), \varphi \in \mathcal{D}\left(Q_{p}\right)\right\}$ со средним

$$
\mathrm{E} \Phi(\varphi)=0
$$

и ковариацией

$$
\operatorname{E} \Phi(\varphi) \bar{\Phi}(\psi)=\int_{Q_{p}} \varphi(t) \bar{\psi}(t) d t
$$

ТЕОрема 3.1. p-Адический бельй шум $\Phi(\varphi ; \omega)$ существует и однозначно определен своим средним и ковариачией.

ДокАЗАТЕЛЬство. Теория меры в линейных топологических пространствах, изложенная, например, в [9], непосредственно применима к нашему случаю.

Если $X$ - локально-выпуклое пространство, то стандартным образом вводится понятие меры цилиндрических множеств в сопряженном пространстве $X^{\prime}$.

Пусть на $X$ задан непрерывный, положительно-определенный, невырожденный билинейньй функционал $B(\varphi, \psi)$. Тогда на $X^{\prime}$ сушествует единственная гауссовская мера цилиндрических множеств $\mu$ такая, что

$$
\int_{X^{\prime}} e^{i\langle\Phi, \varphi\rangle} \mu(\Phi)=e^{-B(\varphi, \varphi) / 2} .
$$

Если $X$ - ядерное пространство, то в силу теоремы 2 работы [9, с. 428] любая гауссовская мера в $X^{\prime}$ является счетно-аддитивной.

В нашем случае мы имеем $X=\mathcal{D}\left(Q_{p}\right)$, и известно [3], что пространство $\mathcal{D}\left(Q_{p}\right)$ является ядерным. Поэтому в силу изложенного существует счетно-аддитивная мера $\mu$ на $\mathcal{D}^{\prime}\left(Q_{p}\right)$ с ковариацией $B(\varphi, \psi)$.

В частности, мы можем взять

$$
B(\varphi, \psi)=\int_{Q_{p}} \varphi(t) \bar{\psi}(t) d t
$$

Теорема доказана. 
Рассмотрим наиболее простую в своей постановке задачу, а именно будем искать $p$-адическое броуновское движение $\left\{\Psi(t ; \omega), t \in Q_{p}\right\}$ как обобщенное решение уравнения типа (2.1) в классе непрерывных функций.

Отметим, что постановка тех или иных граничных задач для обобщенного псевдодифференциального уравнения зависит от его правой части $\Phi$, диктуюшей вместе с оператором $\mathbf{A}$ выбор соответствующего функционального класса для $\Psi$.

Рассмотрим задачу Коши

$$
\begin{gathered}
\mathbf{D}_{t} \Psi=\frac{p}{\sqrt{2(p+1)}} \Phi, \\
\left.\Psi(t ; \omega)\right|_{t=0}=0
\end{gathered}
$$

для почти всех $\omega \in \Omega$. Здесь $\mathbf{D}_{t}$ - оператор Владимирова, $\Phi(\varphi ; \omega)-p$-адический бельй шум $\Phi \in \mathcal{E}^{\prime}$, где $\mathcal{E}^{\prime}$ - пространство обобщенных функций с компактным носителем.

Tеорема 3.2. Решение задачи Коши (3.2), (3.3) существует в классе непрерывных функиий $\mathcal{C}\left(Q_{p}\right)$ для почти всех $\omega \in \Omega$.

ДоказАТЕЛЬСтво. Фундаментальное решение оператора $\mathbf{D}_{t}$ найдено в [3]. Оно имеет вид

$$
\mathcal{E}(t)=-\frac{p-1}{p \ln p} \ln |t|_{p} .
$$

Решение уравнения $(3.2)$ в силу теоремы 1 работы $[1$, с. 173$]$ сушествует в $\mathcal{D}^{\prime}$ и для любого $\omega \in \Omega$ имеет вид

$$
\Psi(t ; \omega)=\frac{p}{\sqrt{2(p+1)}}(\mathcal{E} * \Phi)(t ; \omega)+C(\omega),
$$

где $C(\omega)$ - случайная величина.

С точностью до константы это решение можно формально выразить через фурьеобраз белого шума

$$
\Psi(t ; \omega)=\frac{p}{\sqrt{2(p+1)}} \int_{Q_{p}} \frac{\chi(k t)-1}{|k|_{p}} \widetilde{\Phi}(k ; \omega) d k .
$$

Покажем, что этот несобственный интеграл сушествует в $L^{2}(\Omega, \mathrm{P})$.

Рассмотрим последовательность случайных непрерьвных функций $\left\{\Psi_{n}(t ; \omega)\right\}$ вида

$$
\Psi_{n}(t ; \omega)=\frac{p}{\sqrt{2(p+1)}} \int_{B_{n}} \frac{\chi(k t)-1}{|k|_{p}} \widetilde{\Phi}(k ; \omega) d k,
$$

где $B_{n}=\left\{k \in Q_{p}:|k|_{p} \leqslant p^{n}\right\}$.

Эти функции действительно непрерывны, т.к. фурье-образ $\widetilde{\Phi}(k ; \omega)$ белого шума принадлежит к локально-постоянным функциям $\mathcal{E}\left(Q_{p}\right)$ (см. $[1$, с. 128]), а функция 
$(\chi(k t)-1) /|k|_{p} \in L^{1}\left(B_{n}\right)$, тогда подынтегральная функция в $(3.5)$ принадлежит $L^{1}\left(B_{n}\right)$, а значит, применим аналог теоремы Римана-Лебега [1, с. 127].

Покажем, что последовательность $\left\{\Psi_{n}(t ; \omega)\right\}$ фундаментальна в смысле сходимости в среднем квадратическом.

Из формулы (3.5) получаем

$$
\mathrm{E}\left|\Psi_{n+1}(t)-\Psi_{n}(t)\right|^{2}=\left(\frac{p}{\sqrt{2(p+1)}}\right)^{2} \int_{\Omega}\left|\int_{S_{n+1}} \frac{\chi(k t)-1}{|k|_{p}} \widetilde{\Phi}(k ; \omega) d k\right| \mathrm{P}(d \omega),
$$

где $S_{n+1}=B_{n+1}-B_{n}$. Поменяем порядок интегрирования и проинтегрируем по $\Omega$. Тогда

$$
\begin{aligned}
\mathrm{E}\left|\Psi_{n+1}(t)-\Psi_{n}(t)\right|^{2} & =\left(\frac{p}{\sqrt{2(p+1)}}\right)^{2} \int_{S_{n+1}} \frac{|\chi(k t)-1|^{2}}{|k|_{p}^{2}} d k \leqslant \\
& \leqslant 4\left(\frac{p}{\sqrt{2(p+1)}}\right)^{2} \int_{S_{n+1}} \frac{d k}{|k|_{p}^{2}} .
\end{aligned}
$$

Вычисляя этот $p$-адический интеграл, получаем

$$
\mathrm{E}\left|\Psi_{n+1}(t)-\Psi_{n}(t)\right|^{2} \leqslant \frac{2(p-1)}{p+1} p^{-n},
$$

откуда видно, что в $L^{2}(\Omega, \mathrm{P})$ сушествует предел последовательности $\left\{\Psi_{n}(t ; \omega)\right\}$, который и определяет несобственный интеграл (3.4).

Заметим, что тем самым доказано сушествование интеграла (3.4) и по вероятности.

Докажем теперь, что последовательность непрерывных функций $\left\{\Psi_{n}(t ; \omega)\right\}$ сходится равномерно по $t \in Q_{p}$ для почти всех $\omega \in \Omega$ к функции $\Psi(t ; \omega)$, которая определяется интегралом (3.4). Для этого достаточно выполнить условие

$$
\sum_{n=1}^{\infty} \mathrm{P}\left\{\left|\Psi-\Psi_{n}\right|>\varepsilon\right\}<\infty
$$

для любого $\varepsilon>0$ (см. [10, лемма 2, с. 20]).

Действительно, воспользовавшись неравенством Чебышева

$$
\mathrm{P}\left\{\left|\Psi-\Psi_{n}\right|>\varepsilon\right\} \leqslant \frac{\mathrm{E}\left|\Psi-\Psi_{n}\right|^{2}}{\varepsilon^{2}}
$$

и аналогично тому, как при доказательстве фундаментальности последовательности $\left\{\Psi_{n}(t ; \omega)\right\}$, установив неравенство

$$
\mathrm{E}\left|\Psi-\Psi_{n}\right|^{2} \leqslant \frac{2 p}{p+1} p^{-n}
$$

можно видеть, что условие (3.7) выполняется.

Таким образом, сушествует предел последовательности $\left\{\Psi_{n}(t ; \omega)\right\}$. Он имеет вид (3.4), и последовательность $\left\{\Psi_{n}(t ; \omega)\right\}$ сходится к нему равномерно почти для всех $\omega \in \Omega$. В соответствии с теоремой Лебега предел равномерно сходяшейся последовательности непрерывных функций есть непрерывная функция.

Убедиться в том, что функция (3.4) является решением задачи Коши (3.2), (3.3), можно непосредственной подстановкой. На этом заканчивается доказательство теоремы 3.2 . 
ОПРЕДЕЛЕНИЕ 3.3. p-Адическим броуновским движением на $Q_{p}$ называется гауссовский случайньй процесс $\Psi(t ; \omega), t \in Q_{p}$, такой, что:

1) $\Psi(0 ; \omega)=0$ для почти всех $\omega \in \Omega$;

2) $\mathrm{E} \Psi(t)=0$;

3) $\mathrm{E} \Psi(t) \bar{\Psi}(s)=G_{p}(t, s)=\left(|t|_{p}+|s|_{p}-|s-t|_{p}\right) / 2$.

Теорема 3.3. Решение (3.4) задачи Коши (3.2), (3.3) определяет р-адическое броуновское движение на $Q_{p}$.

ДокАЗАТЕльство. Сушествование решения следует из предыдушей теоремы. Выполнение свойств 1 и 2 определения 3.3 очевидно. Вычислим ковариацию

$\mathrm{E} \Psi(t) \bar{\Psi}(s)=$

$$
=\left(\frac{p}{\sqrt{2(p+1)}}\right)^{2} \int_{\Omega} \mathrm{P}(d \omega) \int_{Q_{p}} d k \frac{\chi(k t)-1}{|k|_{p}} \widetilde{\Phi}(k ; \omega) \int_{Q_{p}} d l \frac{\chi(-l s)-1}{|l|_{p}} \widetilde{\Phi}(l ; \omega) .
$$

Меняя порядок интегрирования (это можно сделать в силу теоремы Фубини), проинтегрируем по $\omega$, а затем по $l$ и получим

$$
\mathrm{E} \Psi(t) \bar{\Psi}(s)=\left(\frac{p}{\sqrt{2(p+1)}}\right)^{2} \int_{Q_{p}} d k \frac{(\chi(k t)-1)(\chi(-k t)-1)}{|k|_{p}^{2}}
$$

Этот интеграл можно представить в виде суммы трех интегралов вида

$$
\int_{Q_{p}} d k \frac{\chi(k t)-1}{|k|_{p}^{2}}=\frac{p+1}{p^{2}}|t|_{p}
$$

(вычисление этого интеграла см. в [11]).

Этим завершается доказательство теоремы.

Приведем некоторые свойства $p$-адического броуновского движения (аналогичные свойства броуновского движения на $Z_{p}$ были получены в [2]):

1. Если $|t|_{p} \neq\left|t^{\prime}\right|_{p}$, то

$$
\mathrm{G}_{p}\left(t, t^{\prime}\right)=\frac{1}{2} \min \left(|t|_{p},\left|t^{\prime}\right|_{p}\right)
$$

2. Дисперсия $p$-адического броуновского движения имеет вид

$$
\mathrm{D}_{p}(h)=\mathrm{E}|\Psi(t+h)-\Psi(t)|^{2}=|h|_{p},
$$

и верна адельная формула

$$
\prod_{p} \mathrm{D}_{p}(h)=1, \quad h \in Q .
$$


3. Если $\left|t_{1}\right|_{p} \leqslant\left|t_{2}\right|_{p}<\left|t_{3}\right|_{p} \leqslant\left|t_{4}\right|_{p}$, то случайный вектор

$$
\left(\Psi\left(t_{2} ; \omega\right)-\Psi\left(t_{1} ; \omega\right), \Psi\left(t_{4} ; \omega\right)-\Psi\left(t_{3} ; \omega\right)\right)
$$

распределен по двумерному гауссовскому закону со средним $(0,0)$. Кроме того,

$$
\mathrm{E}\left(\Psi\left(t_{2}\right)-\Psi\left(t_{1}\right)\right)\left(\bar{\Psi}\left(t_{4}\right)-\bar{\Psi}\left(t_{3}\right)\right)=0 .
$$

4. Процессы $\left\{\Psi(t ; \omega), t \in Q_{p} \backslash\{0\}\right\}$ и $\left\{|t|_{p} \Psi\left(t^{-1} ; \omega\right), t \in Q_{p} \backslash\{0\}\right\}$ имеют одно и то же распределение. Действительно,

$$
\begin{gathered}
\mathrm{E}|t|_{p} \Psi\left(t^{-1}\right)=\mathrm{E} \Psi(t)=0 \\
\mathrm{E}|t|_{p}|s|_{p} \Psi\left(t^{-1}\right) \bar{\Psi}\left(s^{-1}\right)=\mathrm{E} \Psi(t) \bar{\Psi}(s) .
\end{gathered}
$$

5. Для фиксированных $а$ и $\lambda$ из $Q_{p}$ каждый из процессов

$$
\begin{array}{ll}
\Psi(t+a ; \omega)-\Psi(a ; \omega), & t \in Q_{p}, \\
|\lambda|_{p}^{-1} \Psi\left(\lambda_{2} t ; \omega\right), & t \in Q_{p},
\end{array}
$$

является $p$-адическим броуновским движением, т.е. имеет среднее, равное нулю, и ковариационную функцию $\mathrm{G}_{p}(s, t)$.

6. Для ковариационной функции $p$-адического броуновского движения верны формулы

$$
\begin{gathered}
\mathrm{G}_{p}(r s, r t)=|r|_{p} \mathrm{G}_{p}(s, t), \quad r, s, t \in Q_{p} \\
\mathbf{D}_{s} \mathbf{D}_{t} \mathrm{G}_{p}(s, t)=\mathbf{D}_{t} \mathbf{D}_{s} \mathrm{G}_{p}(s, t)=\frac{p^{2}}{2(p+1)} \delta(s-t),
\end{gathered}
$$

где $\mathbf{D}_{t}$ - оператор Владимирова.

7. Найдем переходную функцию $p$-адического броуновского движения. Для упрощения записи будем рассматривать вешественнозначное $p$-адическое броуновское движение. Комплекснозначное $p$-адическое броуновское движение представимо в виде

$$
\Psi(t ; \omega)=X(t ; \omega)+i Y(t ; \omega)
$$

где $X(t ; \omega)$ и $Y(t ; \omega)$ - независимые случайные процессы с нулевыми средними $\mathrm{E} X(t)=$ $\mathrm{E} Y(t)=0$.

Нетрудно показать, что

$$
\mathrm{E} X(t) X(s)=\mathrm{E} Y(t) Y(s)=\mathrm{G}_{p}(s, t) .
$$


Поэтому $n$-мерная плотность вероятности вешественного процесса имеет вид

$$
\mathrm{p}_{t_{1} \ldots t_{n}}=\frac{(\operatorname{det} \Lambda)^{\frac{1}{2}}}{(2 \pi)^{\frac{n}{2}}} \exp \left[-\frac{1}{2} \sum_{i, j=1}^{n} \Lambda\left(t_{i}, t_{j}\right) x_{i} x_{j}\right]
$$

где $\Lambda$ - матрица, обратная к матрице с элементами $\mathrm{G}_{p}\left(t_{i}, t_{j}\right)$. Следовательно, двумерная плотность вероятности $p$-адического броуновского движения имеет вид

$$
\mathrm{p}_{t s}(x, y)=\frac{1}{\left(2 \pi|t|_{p}\right)^{\frac{1}{2}}\left(2 \pi\left(|s|_{p}-g_{p}(s, t)|t|_{p}\right)\right)^{\frac{1}{2}}} \exp \left[-\frac{x^{2}}{2|t|_{p}}-\frac{\left(y-g_{p}(s, t) x\right)^{2}}{2\left(|s|_{p}-g_{p}(s, t)|t|_{p}\right)}\right]
$$

где

$$
g_{p}(s, t)=\frac{\mathrm{G}_{p}(s, t)}{|t|_{p}}
$$

Пользуясь определением плотности условной вероятности, получим плотность переходной функции

$$
p(t, x ; s, y)=\frac{\mathrm{p}_{\xi_{t} \xi_{s}}(x, y)}{\mathrm{p}_{\xi_{t}}(x)}=\frac{1}{\left(2 \pi\left(|s|_{p}-g_{p}^{2}(s, t)|t|_{p}\right)\right)^{\frac{1}{2}}} \exp \left[-\frac{\left(y-g_{p}(s, t) x\right)^{2}}{2\left(|s|_{p}-g_{p}^{2}(s, t)|t|_{p}\right)}\right]
$$

8. Рассмотрим оператор $\mathbf{G}$ на $\mathcal{D}\left(Q_{p}\right)$, который определяется формулой

$$
(\mathbf{G} \varphi)(t)=\int_{Q_{p}} \mathrm{G}_{p}(t, s) \varphi(s) d s
$$

где $\mathrm{G}_{p}(t, s)$ - ковариационная функция $p$-адического броуновского движения.

На множестве функций

$$
\varphi \in \mathcal{D}: \quad \int_{Q p} \varphi(t) d t=0
$$

оператор $\mathbf{G}$ с точностью до множителя $1 / 2$ совпадает с оператором Владимирова - аналогом первообразной $\mathbf{D}^{-2}$ такой, что

$$
\mathbf{D}^{2} \cdot \mathbf{D}^{-2}=\mathbf{1}
$$

(подробнее см. в [1]). 


\section{4. СТОХАСТИЧЕСКИЕ ПСЕВДОДИФФЕРЕНЦИАЛЬНЫЕ УРАВНЕНИЯ НА $Q_{p} \times Q_{p}$}

В этом разделе исследуются граничные задачи для стохастических уравнений вида

$$
\mathbf{A} \Psi=\Phi
$$

с различными псевдодифференциальными операторами $\mathbf{A}$, действующими на функции двух $p$-адических аргументов. Мы рассмотрим $p$-адические стохастические уравнения, аналогичные некоторым основным уравнениям математической физики [12].

Сначала рассмотрим уравнение, решением которого является случайная функция двух переменных. В дальнейшем она называется случайным полем, которое является p-адическим аналогом броуновского листа [13].

Пусть имеем обобщенное стохастическое псевдодифференциальное уравнение

$$
\mathbf{D}_{x} \mathbf{D}_{y} u(x, y ; \omega)=f(x, y ; \omega)
$$

- аналог уравнения

$$
\frac{\partial^{2}}{\partial x \partial y} u(x, y)=f(x, y)
$$

В уравнении (4.1) $x, y \in Q_{p}^{2}, \quad \mathbf{D}_{x}$ - псевдодифференциальный оператор Владимирова, $f(x, y ; \omega)-p$-адический белый шум, т.е. гауссовское обобщенное случайное поле $\{f(\varphi ; \omega)\}$ со средним $\mathrm{E}\langle f, \varphi\rangle=0$ и ковариацией

$$
\mathrm{E}\langle f, \varphi\rangle\left\langle f, \varphi^{\prime}\right\rangle=\int_{Q_{p}^{2}} \varphi(x, y) \bar{\varphi}^{\prime}(x, y) d x d y
$$

Доказательство сушествования $f(x, y ; \omega)$ такое же, как и для белого шума, определенного в разделе 3 .

Обобшенное решение $u(x, y ; \omega)$ уравнения (4.1) будем искать в классе непрерывных функций $\mathcal{C}\left(Q_{p}^{2}\right)$. Для этого уравнения рассмотрим задачу Гурса с граничными условиями

$$
\left.u(x, y ; \omega)\right|_{x=0}=0,\left.\quad u(x, y ; \omega)\right|_{y=0}=0
$$

и при том, что $f(x, y ; \omega) \in \mathcal{E}^{\prime}\left(Q_{p}^{2}\right)$ - обобшенная функция с компактным носителем.

Тогда справедлива следуюшая теорема.

Теорема 4.1. Решение задачи Гурса для стохастического уравнения (4.1) существует в классе непрерывных функиий и единственно для почти всех $\omega \in \Omega$. 
ДокАЗАТЕЛЬСтво. Нетрудно проверить, что фундаментальное решение для оператора $\mathbf{D}_{x} \mathbf{D}_{y}$, удовлетворяющее уравнению

$$
\mathbf{D}_{x} \mathbf{D}_{y} \mathcal{E}(x, y)=\delta(x, y)
$$

имеет вид

$$
\mathcal{E}(x, y)=\left(\frac{p-1}{p \ln p}\right)^{2} \ln |x|_{p} \ln |y|_{p}
$$

Фундаментальное решение не единственно, оно определяется с точностью до слагаемого, являюшегося решением соответствуюшего однородного уравнения.

Решение уравнения (4.1) в $\mathcal{D}^{\prime}\left(Q_{p}^{2}\right)$ можно записать в виде

$u(x, y ; \omega)=\left(\frac{p-1}{p \ln p}\right)^{2} \int_{Q_{p}^{2}} \ln \left|x-x^{\prime}\right|_{p} \ln \left|y-y^{\prime}\right|_{p} f\left(x^{\prime}, y^{\prime} ; \omega\right) d x^{\prime} d y^{\prime}+C_{1}(x ; \omega)+C_{2}(y ; \omega)$ для любых $\omega \in \Omega$.

Подчиняя это решение граничным условиям (4.3) и выражая его через преобразование Фурье белого шума $f$, получаем

$$
u(x, y ; \omega)=\int_{Q_{p}^{2}} \frac{(\chi(-k x)-1)(\chi(-l y)-1)}{|k|_{p}|l|_{p}} \tilde{f}(k, l ; \omega) d k d l
$$

Используя рассуждения, аналогичные рассуждениям в теореме 3.2 , мы устанавливаем, что решение (4.4) непрерывно для почти всех $\omega \in \Omega$. Теорема 4.1 доказана.

Решение (4.4) задачи Гурса для псевдодифференциального стохастического уравнения (4.1) определяет гауссовское случайное поле

$$
\left\{u(x, y ; \omega), x, y \in Q_{p}^{2}\right\}
$$

со средним

$$
\mathrm{E} u(x, y)=0
$$

и с ковариацией, которая имеет факторизованный вид

$$
\mathrm{E} u(x, y) \bar{u}\left(x^{\prime}, y^{\prime}\right)=\frac{p+1}{p^{2}}\left(|x|_{p}+\left|x^{\prime}\right|_{p}-\left|x-x^{\prime}\right|_{p}\right)\left(|y|_{p}+\left|y^{\prime}\right|_{p}-\left|y-y^{\prime}\right|_{p}\right)
$$

Назовем это поле $p$-адическим броуновским листом. 
ЗАмЕчАниЕ. Введем оператор $\square_{p}$ (аналог гиперболического оператора), который определяется формулой

$$
\left(\square_{p} \varphi\right)(r, t)=\int_{Q_{p}^{2}} d u d v\left|u^{2}-v^{2}\right|_{p} \widetilde{\varphi}(u, v) \chi(-u r) \chi(-v t)
$$

где

$$
\widetilde{\varphi}(u, v)=\int_{Q_{p}^{2}} d r d t \varphi(r, t) \chi(u r) \chi(v t)
$$

Уравнение

$$
\left(\square_{p} \varphi\right)(r, t)=f(r, t)
$$

можно понимать как аналог волнового уравнения. Для $p \neq 2$ это уравнение в ограниченной его характеристиками $u= \pm v$ области определяет случайное гауссовское поле как единственное решение, которое совпадает с решением задачи Гурса для уравнения (4.1) с нулевыми данными на характеристиках.

Действительно, если перейти к новым переменным

$$
k=\frac{1}{2}(u+v), \quad l=\frac{1}{2}(u-v),
$$

то оператор $\square_{p}$ переходит в оператор $\mathbf{D}_{x} \mathbf{D}_{y}$.

Обратимся теперь к стохастическому уравнению

$$
\Delta_{p} \psi(x, y ; \omega)=f(x, y ; \omega)
$$

с псевдодифференциальным оператором ( $p$-адический аналог оператора Лапласа), который определяется формулой

$$
\left(\Delta_{p} \psi\right)(x, y)=\int_{Q_{p}^{2}} d k d l\left|k^{2}+l^{2}\right|_{p} \tilde{\psi}(k, l) \chi(-k x) \chi(-l y)
$$

Будем рассматривать отдельно два случая, которые связаны с существованием корней алгебраического уравнения

$$
k^{2}+l^{2}=0
$$

Это уравнение для $p \equiv 3(\bmod 4)$ имеет лишь тривиальное решение $k=0, l=0$ над полем $p$-адических чисел, а для $p \equiv 1(\bmod 4)$, кроме того, и нетривиальные решения $k= \pm q l, q^{2}=-1: q \in Q_{p}$.

1. Пусть $p \equiv 1(\bmod 4)$. Построим для этого случая фундаментальное решение оператора (4.8). Рассмотрим уравнение

$$
\Delta_{p} \mathcal{E}(x, y)=\delta(x, y)
$$


ПРЕДЛОЖЕНИЕ 4.1. Функиия

$$
\mathcal{E}(x, y)=\left(\frac{p-1}{p \ln p}\right)^{2} \ln |x+q y|_{p} \ln |x-q y|_{p}
$$

является фундаментальным решением оператора $\Delta_{p}$.

ДокАЗАТЕльство. Это предложение доказывается непосредственной подстановкой функции (4.10) в уравнение (4.9).

Рассмотрим теперь стохастическое уравнение (4.7) и сделаем замену переменных

$$
z=x+q y, \quad \bar{z}=x-q y
$$

Тогда

$$
\begin{aligned}
\Delta_{p} \psi(x, y ; \omega) & \rightarrow \mathbf{D}_{z} \mathbf{D}_{\bar{z}} \varphi(z, \bar{z} ; \omega), \\
f(x, y ; \omega) \rightarrow g(z, \bar{z} ; \omega) & =f\left(\frac{1}{2}(z+\bar{z}), \frac{1}{2 q}(z-\bar{z}) ; \omega\right), \\
\psi(x, y ; \omega) \rightarrow \varphi(z, \bar{z} ; \omega) & =\psi\left(\frac{1}{2}(z+\bar{z}), \frac{1}{2 q}(z-\bar{z}) ; \omega\right)
\end{aligned}
$$

Уравнение (4.7) примет вид

$$
\mathbf{D}_{z} \mathbf{D}_{\bar{z}} \varphi(z, \bar{z} ; \omega)=g(z, \bar{z} ; \omega)
$$

Для граничных условий

$$
\left.\varphi(z, \bar{z} ; \omega)\right|_{z=0}=0,\left.\quad \varphi(z, \bar{z} ; \omega)\right|_{\bar{z}=0}=0
$$

задача сводится к рассмотренной выше задаче Гурса.

2. Пусть $p \equiv 3(\bmod 4)$. Построим фундаментальное решение оператора $(4.8)$. Рассмотрим уравнение

$$
\Delta_{p} \mathcal{E}(x, y)=\delta(x, y)
$$

ПРЕДЛОЖЕНИЕ 4.2. Обобщенная функиия

$$
\mathcal{E}(x, y)=-\frac{p^{2}-1}{p^{2} \ln p^{2}} \ln \left|x^{2}+y^{2}\right|_{p}
$$

является фундаментальным решением оператора $\Delta_{p} \partial$ яя $\equiv 3(\bmod 4)$. 
ДокАЗАтЕльство. Преобразование Фурье функции $\ln \left|x^{2}+y^{2}\right|_{p}$ имеет вид

$$
F\left[\ln \left|x^{2}+y^{2}\right|_{p}\right](k, l)=-\frac{p^{2} \ln p^{2}}{p^{2}-1}\left|k^{2}+l^{2}\right|_{p}^{-1}
$$

Поэтому для любой функции $\varphi(x, y) \in \mathcal{D}\left(Q_{p}^{2}\right)$ в силу определения оператора $\Delta_{p}$ имеем

$$
\left\langle\Delta_{p} \mathcal{E}(x, y), \varphi(x, y)\right\rangle=\left\langle\int_{Q_{p}^{2}} \chi(-k x) \chi(-l y), \varphi(x, y) d k d l\right\rangle=\langle\delta(x, y), \varphi(x, y)\rangle
$$

что и требовалось доказать.

Рассмотрим стохастическое псевдодифференциальное уравнение с оператором $\Delta_{p}$ при $p \equiv 3(\bmod 4)($ уравнение эллиптического типа)

$$
\begin{aligned}
& \Delta_{p} \psi(x, y ; \omega)=f(x, y ; \omega), \\
& \left.\psi(x, y ; \omega)\right|_{x=0, y=0}=0
\end{aligned}
$$

для любых $\omega \in \Omega$. Напомним, что $f(x, y ; \omega)$ - это $p$-адический белый шум, определенный в начале раздела 4 .

Теорема 4.2. Если $f(x, y ; \omega) \in \mathcal{E}^{\prime}\left(Q_{p}^{2}\right)$, то решение задачи (4.14), (4.15) существует в классе непрерывных функций $\mathcal{C}\left(Q_{p}^{2}\right)$ для почти всех $\omega \in \Omega$.

ДоказАтельство. Решение будем искать в $\mathcal{D}^{\prime}$ в виде

$$
\psi(x, y ; \omega)=\mathcal{E}(x, y) * f(x, y ; \omega)+C(\omega)
$$

где $C(\omega)$ - случайная величина. Подставляя сюда выражение для фундаментального решения (4.13), получим

$$
\psi(x, y ; \omega)=-\frac{p^{2}-1}{p^{2} \ln p^{2}} \int_{Q_{p}^{2}} \ln \left|\left(x-x^{\prime}\right)^{2}+\left(y-y^{\prime}\right)^{2}\right|_{p} f\left(x^{\prime}, y^{\prime} ; \omega\right) d x^{\prime} d y^{\prime}+C(\omega)
$$

С точностью до константы это решение выражается через преобразование Фурье белого шума следуюшим образом:

$$
\psi(x, y ; \omega)=\int_{Q_{p}^{2}} \frac{\chi(-(k x+l y))-1}{\left|k^{2}+l^{2}\right|_{p}} \tilde{f}(k, l ; \omega) d k d l .
$$

Этот несобственный интеграл определен в $L^{2}(\Omega, \mathrm{P})$. Далее, проводя аналогичные рассуждения, что и в теореме 3.2 , доказываем непрерьвность решения (4.16) для почти всех $\omega \in \Omega$. Нетрудно убедиться, что (4.16) удовлетворяет (4.14), (4.15). Доказательство теоремы 4.2 закончено. 
Стохастическая функция (4.16) определяет гауссовское случайное поле $\{\psi(z ; \omega)$, $\left.z \in Q_{p}^{2}\right\}$, которое будем назьвать $p$-адическим броуновским движением Леви.

Среднее случайного поля $\psi$ равно нулю:

$$
\mathrm{E} \psi(z ; \omega)=0
$$

где $z=\left(x_{1}, x_{2}\right) \in Q_{p}^{2}$, что непосредственно вытекает из (4.16).

Ковариация случайного поля $\psi$ имеет вид

$$
\mathrm{E} \psi(z ; \omega) \bar{\psi}\left(z^{\prime} ; \omega\right)=\frac{p^{2}+1}{p^{4}}\left(|(z, z)|_{p}+\left|\left(z^{\prime}, z^{\prime}\right)\right|_{p}-\left|\left(z-z^{\prime}, z-z^{\prime}\right)\right|_{p}\right) .
$$

Мы не будем приводить подробного вычисления ковариации, отметив лишь, что после интегрирования по $\omega$ задача сведется к вычислению интегралов вида

$$
\int_{Q_{p}^{2}} d^{2} w \frac{\chi((w, z))}{|(w, w)|_{p}^{2}}
$$

По поводу вычисления таких интегралов см. [1] и [11] .

Благодарности. Автор признателен И. В. Воловичу за помощь в работе над статьей, а также В. С. Владимирову и С. В. Козыреву за черезвычайно полезные советы.

Работа была поддержана грантом INTAS № 96-06-98.

\section{Список литературы}

[1] В. С. Владимиров, И. В. Волович, Е. И. Зеленов. р-Адический анализ и математическая физика. М.: Наука, 1994.

[2] А. Х. Бикулов, И. В. Волович. Изв. РАН. Сер. матем. 1997. Т. 61. № 3. С. 75-90.

[3] В. С. Владимиров. УМН. 1988. Т. 43. № 5. С. 17-53.

[4] S. Albeverio, W. Karwowski. Stoch. Proc. Appl. 1994. V. 53. P. 1-22.

[5] W. Karwowski, R. Vilela Mendes. J. Math. Phys. 1994. V. 35. P. 4637-4650.

[6] M. D. Missarov. Ann. Inst. Henri Poincaré. 1989. V. 50. P. 357-367.

[7] A. N. Kochubei. Potential Analysis. 1997. V. 6. P. 105-125.

[8] А. Н. Кочубей. Изв. АН. Сер. матем. 1992. Т. 39. С. 1263-1280.

[9] И. А. Гельфанд, Н. Я. Виленкин. Некоторые применения гармонического анализа. Оснащенные гильбертовы пространства. Обобщенные функции. Т. 4. М.: Физматгиз, 1961.

[10] И. И. Гихман, А. В. Скороход. Теория случайных процессов. Т. 1. М.: Наука, 1971.

[11] А. Х. Бикулов. ТМФ. 1991. Т. 87. № 3. С. 376-390.

[12] В. С. Владимиров. Уравнения математической физики. М.: Наука, 1988.

[13] Ю. А. Розанов. Случайные поля и стохастические уравнения с частными производными. М.: Наука, 1995. 